\title{
A note on the concept of acoustic center
}

\author{
Jacobsen, Finn; Barrera Figueroa, Salvador; Rasmussen, Knud
}

Published in:

Journal of the Acoustical Society of America

Link to article, DOI:

$10.1121 / 1.1652036$

Publication date:

2004

Document Version

Publisher's PDF, also known as Version of record

Link back to DTU Orbit

Citation (APA):

Jacobsen, F., Barrera Figueroa, S., \& Rasmussen, K. (2004). A note on the concept of acoustic center. Journal of the Acoustical Society of America, 115, 1468-1473. https://doi.org/10.1121/1.1652036

\section{General rights}

Copyright and moral rights for the publications made accessible in the public portal are retained by the authors and/or other copyright owners and it is a condition of accessing publications that users recognise and abide by the legal requirements associated with these rights.

- Users may download and print one copy of any publication from the public portal for the purpose of private study or research.

- You may not further distribute the material or use it for any profit-making activity or commercial gain

- You may freely distribute the URL identifying the publication in the public portal

If you believe that this document breaches copyright please contact us providing details, and we will remove access to the work immediately and investigate your claim 


\title{
A note on the concept of acoustic center ${ }^{\text {a) }}$
}

\author{
Finn Jacobsen, ${ }^{\text {b) }}$ Salvador Barrera Figueroa, ${ }^{\text {c) }}$ and Knud Rasmussen ${ }^{\text {d) }}$ \\ Acoustic Technology, Ørsted•DTU, Technical University of Denmark, Building 352, \\ DK-2800, Kgs. Lyngby, Denmark
}

(Received 1 August 2003; revised 2 January 2004; accepted 13 January 2004)

\begin{abstract}
The acoustic center of a reciprocal transducer is defined as the point from which spherical waves seem to be diverging when the transducer is acting as a source. This paper examines various ways of determining the acoustic center of a source, including methods based on deviations from the inverse distance law and methods based on the phase response. The considerations are illustrated by experimental results for condenser microphones. (c) 2004 Acoustical Society of America.
\end{abstract}

[DOI: $10.1121 / 1.1652036]$

PACS numbers: 43.20.Rz, 43.38.Kb, 43.58.Vb [AJZ]

Pages: $1468-1473$

\section{INTRODUCTION}

Any finite source of sound generates a sound pressure that, in any direction, sufficiently far away under free-field conditions varies in inverse proportion to the distance from the source. ${ }^{1}$ Very far away the exact position from which this inverse distance law applies is obviously not very important, but closer to the source the position of this "acoustic center" may be of some concern.

The concept of acoustic center is frequently referred to in the literature. It is defined in Refs. 2 and 3 as the position of the point from which spherical wavefronts appear to diverge, and in Refs. 4 and 5 as the position from which the sound pressure varies inversely as the distance. Knowledge of the acoustic center is of concern whenever a well-defined distance to a source is needed, for example, in testing anechoic rooms by measuring deviations from the inverse distance law. ${ }^{6,7}$ It can also be important to know the acoustic center of a transducer that is used primarily as a receiver. Most measurement microphones are used under free-field conditions, and free-field reciprocity calibration is the most accurate method of determining the free-field sensitivity of microphones. This method involves measuring the transfer function between pairs of microphones., ${ }^{3,8,9}$ The "acoustic distance" between the transmitter and receiver microphone must be known, and since the two transducers cannot be far from each other because of the extremely poor signal-tonoise ratio of the measurement, their acoustic centers must be known with great accuracy. The acoustic center of a reciprocal transducer does not depend on whether it is used as receiver or transmitter. ${ }^{10-12}$

In general the acoustic center of a source varies with the frequency, with the direction of the observer, and with the distance from the source, ${ }^{2}$ as demonstrated theoretically in Refs. 13 and 14. However, Rasmussen showed that the de-

\footnotetext{
${ }^{a)}$ Portions of this work were presented in "A note on the acoustic centre of condenser microphones," Proceedings of Tenth International Congress on Sound and Vibration, Stockholm, Sweden, July 2003.

${ }^{b)}$ Author to whom correspondence should be addressed; Electronic mail: fja@oersted.dtu.dk

c)Electronic mail: sbf@oersted.dtu.dk

${ }^{\mathrm{d})}$ Electronic mail: kr@oersted.dtu.dk
}

pendence of the distance can be ignored in reciprocity calibration of microphones. ${ }^{14}$

Most results presented in the literature have been determined from deviations between the amplitude (or rms value) of the sound pressure and the inverse distance law. Cox measured the acoustic centers of various transducers using a dismantled lathe bed for positioning the scanning microphone and a 10-turn potentiometer for compensating for the $1 / r$ dependence. ${ }^{13}$ Rasmussen measured the acoustic centers of microphones of type LS1 (laboratory standard "1 in.") with and without protection grid. ${ }^{14}$ The results, which were found to agree reasonably well with approximate theoretical considerations, are the basis of the values given in the IEC standard from $1974^{15}$ and the slightly modified values given in the newer one. ${ }^{3}$ Rasmussen's data were deduced from the decay of the sound pressure generated by a microphone with the distance, measured with a probe microphone and recorded with a level recorder. In a paper primarily concerned with measuring the air attenuation Hruska and Koidan described a procedure based on fitting a second-order polynomial in the distance between two transducers to an expression based on the distance and the magnitude of the transfer function between them. ${ }^{16}$ More recently Rasmussen and Sandermann Olsen summarized the results of a comparison of acoustic center values among several European laboratories. ${ }^{17}$ Juhl has determined the acoustic centers of microphones of types LS1 and LS2 (laboratory standard " 0.5 in.") by calculating the sound pressure amplitude at positions on the microphone axes using the boundary value method and assuming a parabolic movement of the diaphragms. ${ }^{18}$ And finally Wagner and Nedzelnitsky have determined the acoustic center of microphones of type LS2 using measured values of the magnitude of the transfer function between two microphones. ${ }^{19}$

A few examples of methods of determining the acoustic center from phase measurements have been found in the literature. Ando determined the acoustic center of a "pipe horn" loudspeaker from measurements of the phase shift between two positions. ${ }^{20}$ Rasmussen also attempted to determine spherical wavefronts and thus acoustic centers from phase measurements. ${ }^{14}$ However, this method was found to fail completely because of imperfections of the anechoic 
room-although the anechoic room used in these measurements is a very good one. ${ }^{4}$ Trott proposed a definition of the acoustic center based on phase considerations and presented some theoretical and numerical results, ${ }^{21}$ and Vorländer and Bietz described a method of determining the acoustic center from the group delay of the complex sensitivity of a microphone and presented some experimental results. ${ }^{22}$

The purpose of this paper is to examine and discuss the concept of acoustic center and to present some experimental results for condenser microphones.

\section{THE CONCEPT OF ACOUSTIC CENTER}

Both definitions of the acoustic center, "the position from which outgoing wavefronts appear to diverge in the far field" $" 2,3$ and "the position from which the sound pressure varies inversely as distance," 4,5 seem to imply the existence of an equivalent point source. The idea of replacing a real, extended source by an equivalent point source seems to be straightforward, and this apparent simplicity perhaps explains why most authors have taken the concept for granted. In fact the only fundamental considerations found in the literature are the Cox and Rasmussen theoretical analyses ${ }^{13,14}$ and Trott's "redefinition.",21

For simplicity, the following discussion is restricted to axisymmetric sources observed from positions on the axis. In this case the acoustic center must be somewhere on the axis. Let $r$ indicate the physical distance from the observation point to a point on the real source, and let $x$ indicate the position of the acoustic center. Several approaches are now possible.

(i) If an analytical expression of the sound field generated by the source is available then one can adjust the parameter $x$ in the expression of the amplitude of the sound pressure generated by the equivalent source,

$$
\left|p_{\mathrm{eq}}(r)\right|=\frac{\rho c k Q_{\mathrm{eq}}}{4 \pi(r-x)},
$$

to the amplitude of the pressure generated by the source under investigation $p(r)$ until the two expressions agree. (Here $k$ is the wave number, $\rho c$ is the characteristic impedance of air, and $Q_{\text {eq }}$ is the volume velocity of the equivalent source, determined from far field considerations in the direction of concern.) The result is in general a function of $k$ and $r$,

$$
x(k, r)=r-\frac{\rho c k Q_{\mathrm{eq}}}{4 \pi|p(r)|} .
$$

Cox and Rasmussen used this method in the theoretical part of their studies. ${ }^{13,14}$

(ii) One can plot the reciprocal sound pressure amplitude as a function of $r$, fit a straight line over the region of concern, and determine its intersection with the $r$ axis, as specified in Ref. 23. This corresponds to the following expression for the position of the acoustic center,

$$
\begin{aligned}
x(k, r) & =r-\frac{1}{|p(r)|} / \frac{\partial(1 /|p(r)|)}{\partial r} \\
& =r+|p(r)| / \frac{\partial|p(r)|}{\partial r}
\end{aligned}
$$

where the rate of change in practice (where no analytical expression is available) must be estimated over a suitable interval. This method was used by Cox and Rasmussen in their experimental work, ${ }^{13,14}$ and by Juhl in his numerical study. ${ }^{18}$ Similar methods based on measured transfer functions between pairs of microphones have been used in the work reported in Refs. 17, 19, and 24.

(iii) One can estimate the position of the acoustic center from the phase response corrected for the phase shift associated with the distance to the observation point. ${ }^{21}$ This corresponds to equating the phase of the sound pressure generated by the equivalent point source with the phase of the sound pressure generated by the source under investigation. Evidently, a phase reference, for example, the velocity at some position on the source $U$, is needed. The result is the expression

$$
x(k, r)=\frac{1}{k} \operatorname{Arctan}\left(\frac{\operatorname{Im}\left\{\left(p(r) e^{j k r} / j U e^{j \omega t}\right)\right\}}{\operatorname{Re}\left\{\left(p(r) e^{j k r} / j U e^{j \omega t}\right)\right\}}\right) .
$$

Note that the $e^{j \omega t}$ sign convention is used in this paper.

(iv) Alternatively, one can use the corresponding group delay multiplied by the speed of sound, ${ }^{22}$

$$
x(k, r)=\frac{\partial}{\partial k}\left(\operatorname{Arctan}\left(\frac{\operatorname{Im}\left\{\left(p(r) e^{j k r} / j U e^{j \omega t}\right)\right\}}{\operatorname{Re}\left\{\left(p(r) e^{j k r} / j U e^{j \omega t}\right)\right\}}\right)\right) .
$$

(v) One can estimate the position of the center from the curvature of wavefronts determined from phase measurements. ${ }^{14,20}$

\section{A. Some simple examples}

A few examples will be presented to demonstrate how the methods work. The first source to be studied is a pulsating sphere. The sound pressure a distance $r$ from the center of a pulsating sphere with radius $a$ and vibrational velocity $U$ is 1

$$
\begin{aligned}
p(r) & =\frac{j \rho c k a^{2} U}{r(1+j k a)} e^{j(\omega t-k(r-a))} \\
& =\frac{j \rho c k a^{2} U}{r \sqrt{1+(k a)^{2}}} e^{j(\omega t-k(r-a)-\operatorname{Arctan}(k a))} .
\end{aligned}
$$

It is apparent that the volume velocity of the equivalent monopole that gives the same sound pressure in the far field is

$$
Q_{\mathrm{eq}}=\frac{4 \pi a^{2} U}{\sqrt{1+(k a)^{2}}} .
$$

The two definitions based on amplitude considerations [Eqs. (2) and (3)] give $x(k, r)=0$. In other words, amplitude considerations place the acoustic center of a pulsating sphere in the center of the sphere. By contrast, the two definitions based on phase considerations give 


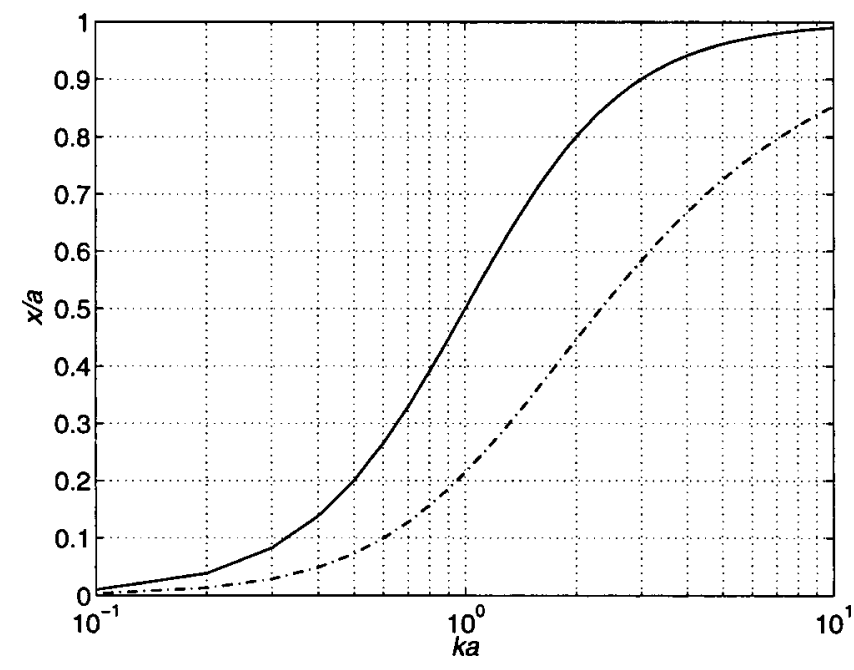

FIG. 1. The acoustic center of a pulsating sphere of radius $a$ calculated using the phase delay [Eq. (8)] (-•-) and the group delay [Eq. (9)] (-).

$$
x(k, r)=a-\frac{1}{k} \operatorname{Arctan} k a
$$

and

$$
x(k, r)=\frac{\partial}{\partial k}(k a-\operatorname{Arctan} k a)=a-\frac{a}{1+(k a)^{2}},
$$

respectively. [Equation (8) agrees with Trott's results. ${ }^{21}$ ] As Fig. 1 shows, the two methods based on phase considerations place the acoustic center of a pulsating sphere in the center of the sphere at low frequencies, but as $k a$ increases it moves towards the surface. This makes sense since this is the point where the phase reference is taken. Note that none of the acoustic centers of the pulsating sphere depends on the distance $r$.

The second example is a baffled circular piston. The sound pressure generated by a vibrating piston with radius $a$ and vibrational velocity $U$ at a position on the axis a distance $r$ from the piston is ${ }^{1}$

$$
\begin{aligned}
p(r) & =\rho c U e^{j \omega t}\left(e^{-j k r}-e^{-j k \sqrt{r^{2}+a^{2}}}\right) \\
& =2 j \rho c U \sin (k \Delta) e^{j(\omega t-k(r+\Delta))},
\end{aligned}
$$

where

$$
\Delta=\frac{1}{2}\left(\sqrt{r^{2}+a^{2}}-r\right) .
$$

Inserting into Eq. (2) using a volume velocity of

$$
Q_{\mathrm{eq}}=2 U \pi a^{2}
$$

gives

$$
x(k, r)=r-\frac{k a^{2}}{4 \sin (k \Delta)} .
$$

The second amplitude-based method, Eq. (3), gives a different expression,
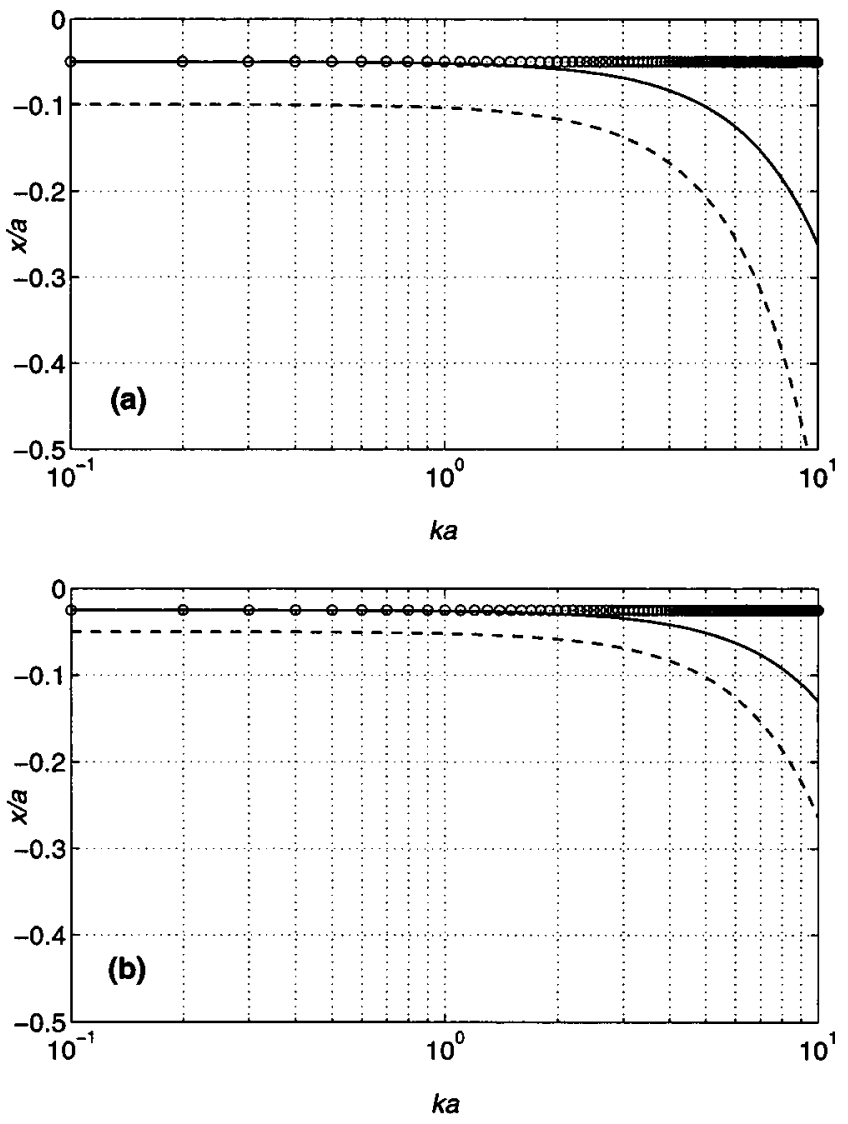

FIG. 2. The acoustic center of a circular piston in a baffle of radius $a$ seen from a distance of (a) five radii and (b) 10 radii. —, First amplitude-based method [Eq. (13)]; --, second amplitude-based method [Eq. (14)]; $\bigcirc$, both phase-based methods [Eq. (15)].

$$
x(k, r)=r-\frac{\tan (k \Delta)}{k \Delta} \sqrt{r^{2}+a^{2}} .
$$

The phase approach based on Eq. (4) gives yet another expression,

$$
x(k, r)=-\Delta=-\frac{1}{2}\left(\sqrt{r^{2}+a^{2}}-r\right) \simeq-\frac{a^{2}}{4 r},
$$

and since the phase of Eq. (10) is a linear function of the frequency the group delay method, Eq. (5), gives the same value in this case.

As can be seen from Fig. 2, the acoustic center given by Eq. (13) is negative (although it can be positive when the normalized frequency $k a$ is very high), its numerical value increases with the frequency (although very weakly if $k a$ $<1$ ), and it is essentially inversely proportional to $r$. (The last mentioned property disagrees with results presented in Rasmussen's report. ${ }^{14}$ ) Equation (14) gives values with the same tendency but approximately twice as large. Equation (15) agrees fairly well with Eq. (13) only when $k a<1$. According to all the methods the acoustic center of a baffled circular piston is placed behind the piston. Unfortunately the position of the center depends not only on the frequency but also on the distance, unlike the acoustic center of a pulsating sphere. 
The third example is that of a point source on a rigid sphere. The sound pressure generated by a monopole with a volume velocity of $Q$ placed on a rigid sphere with radius $a$ is $^{25}$

$p(r)=-\frac{j \rho c Q e^{j \omega t}}{4 \pi a^{2}} \sum_{m=0}^{\infty} \frac{(2 m+1)^{2} h_{m}^{(2)}(k r)}{m h_{m-1}^{(2)}(k a)-(m+1) h_{m+1}^{(2)}(k a)}$,

at a position in front of the monopole a distance $r$ from the center of the sphere. In this expression $h_{m}^{(2)}(x)$ is the spheri- cal Hankel function of the second kind and order $m$. The equivalent monopole can be shown to have a volume velocity of

$$
Q_{\mathrm{eq}}=-\frac{Q}{(k a)^{2}} \sum_{m=0}^{\infty} \frac{(2 m+1)^{2} j^{m+1}}{m h_{m-1}^{(2)}(k a)-(m+1) h_{m+1}^{(2)}(k a)} .
$$

(This expression approaches $Q$ asymptotically at low frequencies.) Inserting into Eq. (2) gives

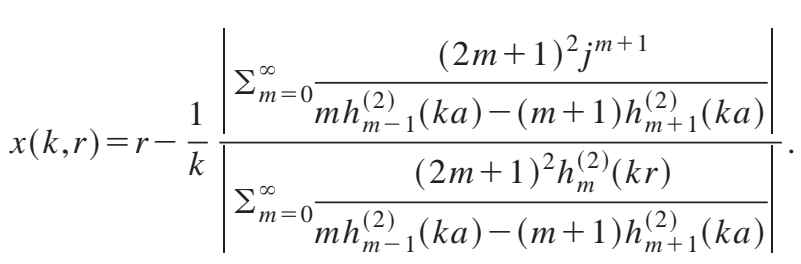

Equation (18) agrees with equations derived by Cox and Rasmussen. ${ }^{13,14}$ The amplitude method based on Eq. (3) gives a somewhat more complicated expression,

$$
x(k, r)=r+2 \frac{\sum_{m=0}^{\infty} \sum_{n=0}^{\infty} \frac{(2 m+1)^{2} h_{m}^{(2)}(k r)(2 n+1)^{2} h_{n}^{(1)}(k r)}{\left(m h_{m-1}^{(2)}(k a)-(m+1) h_{m+1}^{(2)}(k a)\right)\left(n h_{n-1}^{(1)}(k a)-(n+1) h_{n+1}^{(1)}(k a)\right)}}{\sum_{m=0}^{\infty} \sum_{n=0}^{\infty} \frac{(2 m+1)^{2}(2 n+1)^{2}\left(h_{m}^{(2)}(k r) \partial h_{n}^{(1)}(k r) / \partial r+h_{n}^{(1)}(k r) \partial h_{m}^{(2)}(k r) / \partial r\right)}{\left(m h_{m-1}^{(2)}(k a)-(m+1) h_{m+1}^{(2)}(k a)\right)\left(n h_{n-1}^{(1)}(k a)-(n+1) h_{n+1}^{(1)}(k a)\right)}},
$$

in which $h_{m}^{(1)}(x)$ is the spherical Hankel function of the first kind and order $m$, and

$$
\frac{\partial h_{m}(k r)}{\partial r}=\frac{k}{2 m+1}\left(m h_{m-1}(k r)-(m+1) h_{m+1}(k r)\right)
$$

for Hankel functions of either kind. ${ }^{26}$ Finally the method based on the phase delay gives

$$
x(k, r)=\frac{1}{k} \operatorname{Arctan}\left(\frac{\operatorname{Im}\left\{\sum_{m=0}^{\infty} \frac{(2 m+1)^{2} h_{m}^{(2)}(k r) e^{j k r}}{m h_{m-1}^{(2)}(k a)-(m+1) h_{m+1}^{(2)}(k a)}\right\}}{\operatorname{Re}\left\{\sum_{m=0}^{\infty} \frac{(2 m+1)^{2} h_{m}^{(2)}(k r) e^{j k r}}{m h_{m-1}^{(2)}(k a)-(m+1) h_{m+1}^{(2)}(k a)}\right\}}\right) .
$$

Figure 3 shows the acoustic center of the point source on the sphere calculated using Eqs. (18), (19), and (21). It is apparent that the acoustic center based on amplitude considerations is placed in front of the physical source at low frequencies and moves towards the source as the frequency is increased. In fact, Eq. (18) approaches 1.5 times the radius of the sphere as $k a$ goes to zero and $k r$ goes to infinity. ${ }^{13,14}$ By contrast, the phase-based acoustic center is placed behind the entire sphere at low frequencies.

\section{B. Discussion}

In practice it may be difficult to determine the volume velocity of the equivalent source, so the first method, which at first glance seems quite reasonable, may be difficult to use in experimental or numerical work. More importantly, it is not completely obvious how the resulting acoustic center should be interpreted, although it is clear that it reflects de- viations from the inverse distance law of a point source adjusted to give the same far field. The second method, which does not require any knowledge of the source and gives the point from which the inverse distance law appears to apply seen from a certain distance, would seem to be more useful, and this is clearly the relevant method for free-field reciprocity calibration of microphones and for testing anechoic rooms. The method based on the phase delay may well be useful for other applications. It may, for example, be useful to know the position of the phase-related acoustic centers of loudspeaker units. On the other hand it is not clear from Ref. 22 why the group delay, which is a quantity associated with the speed with which the energy of a wave packet travels in a dispersive medium, ${ }^{27,28}$ should be relevant. The method based on the curvature of wavefronts is unlikely to be reliable in practice because of imperfections of the anechoic room. 

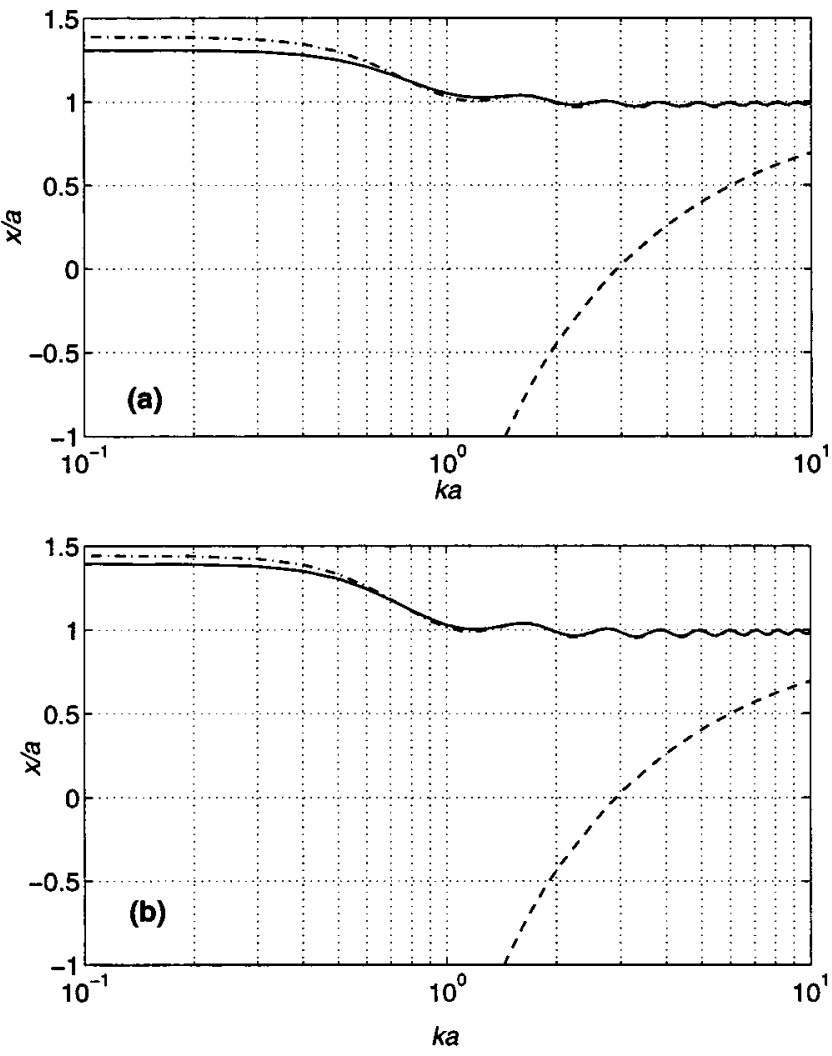

FIG. 3. The acoustic center of a point source on a rigid sphere of radius $a$ seen from a distance of (a) five radii and (b) 10 radii. - •-, First amplitudebased method [Eq. (18)]; —, second amplitude-based method [Eq. (19)]; ,-- phase delay method [Eq. (21)].

One might have hoped that the various methods would lead to the same result, but that seems to be the case only if the source is a point monopole (in free space or on a rigid baffle). The analysis of a few simple cases has demonstrated that the concept of an acoustic center is more complicated than one might have expected.

It is apparent that the acoustic center of a source of sound can be placed behind the physical vibrating surface or in front of it. That amplitude considerations lead to the acoustic center of a baffled piston being placed behind the piston can be explained by the fact that the sound pressure assumes a finite value in front of the piston unless the piston is infinitely small (a point source). In the latter case the center coincides with the position of the source. However, if the baffle is "folded back to form a sphere" 14 the center moves forward at low frequencies, presumably because of interference between the direct wave and the one that has traveled around the sphere. Another explanation is that the sound pressure in all the outgoing waves [the terms of Eq. (16)] except that of zero order decays faster than $1 / r$ near the sphere. Such considerations lead Rasmussen to conclude that the acoustic center of a condenser microphone could be expected to be placed at a position about half the radius in front of the diaphragm at low frequencies, and closer to the diaphragm or even behind it at high frequencies. ${ }^{14}$

\section{EXPERIMENTAL RESULTS}

To supplement the theoretical examples some experiments have been carried out. The electrical transfer functions

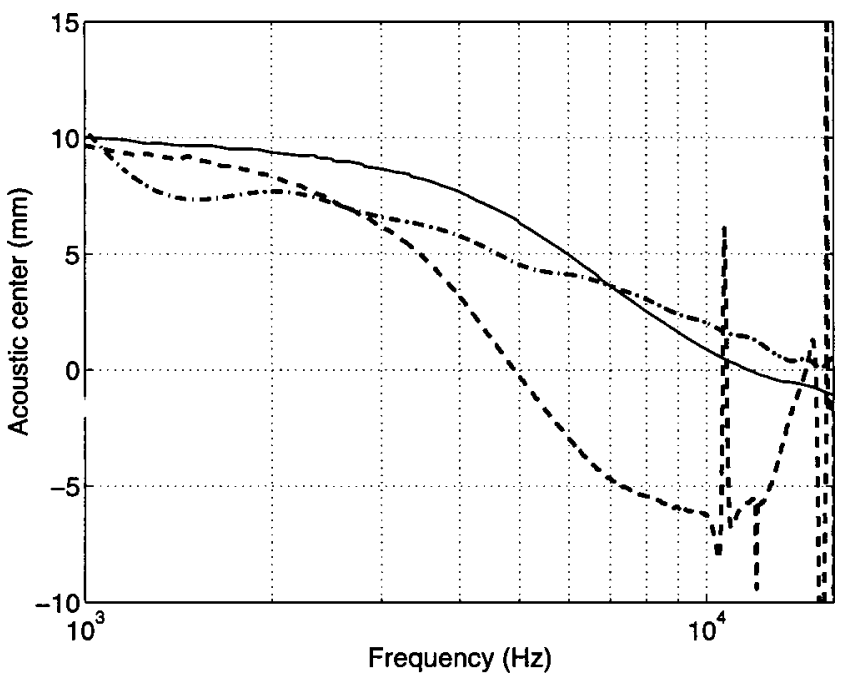

FIG. 4. Acoustic centers of microphones of type LS1 determined from the rate of change of the modulus of the transfer function $\left(-\bullet_{-}\right)$, and determined from the free-field phase response using the phase delay $(-)$ and using the group delay (--). The free-field phase response has been corrected for the phase of the pressure sensitivity.

between three pairs of condenser microphones of type LS1 have been measured at four different distances (from 200 to $500 \mathrm{~mm})$ in a small anechoic room, using a special timeselective technique for removing the influence of cross-talk, reflections from the walls, and standing waves between the microphones. ${ }^{24,29}$ The acoustic centers were determined from the rate of change of the amplitude of these functions (corrected for the absorption of air), using the procedure based on Eq. (3). Figure 4 shows the results, which are in good agreement with values given in the IEC standard ${ }^{3}$ up to about $18 \mathrm{kHz}$. For comparison the results of determining the acoustic center from phase measurements are also shown. In this case the physical distance between the two microphone diaphragms has been used in calculating the free-field sensitivities from measured transfer functions. The resulting complex free-field sensitivities have been corrected for the phase of the pressure sensitivities and finally processed as indicated by Eqs. (4) and (5). (The phase of the pressure sensitivity is related to the internal mechanism of the microphone and has nothing to do with the phase resulting from the geometry and the vibrational pattern of the diaphragm.) The spikes in the curve determined from the group delays are due to the differentiation. It is apparent that neither the results deduced from the phase delay nor the corresponding values determined from the group delay are in agreement with the acoustic center determined from amplitude measurements. However, it is clear from the analysis above that no such agreement could be expected.

Using the physical distance between the microphones in determining their free-field sensitivities from measured transfer functions has the effect of making the sensitivities slightly dependent on the distance. When the distance between the amplitude-based acoustic centers is used in the calculations the resulting free-field sensitivities become essentially independent of the distance, and it is obviously these centers that should be used. The amplitude-based acoustic centers do not depend appreciably on the distance 
unless the microphones are much closer to each other than the minimum distance used in these measurements.

\section{CONCLUSIONS}

The idea of replacing a real, extended source by an equivalent point source from which outgoing wavefronts appear to diverge is deceptively simple, and various procedures for determining the position of such a source give in general different results. The most useful approach, in reciprocity calibration of transducers as well as in testing the quality of anechoic rooms, would seem to be the one that gives the position from which the inverse distance law applies, as seen from positions in the region of concern.

${ }^{1}$ A. D. Pierce, Acoustics. An Introduction to Its Physical Principles and Applications (The American Heritage of Physics, New York, 1989). See Secs. 4.1, 4.5, and 5.7.

${ }^{2}$ C. L. Morfey, Dictionary of Acoustics (Academic, San Diego, 2001).

${ }^{3}$ IEC International Standard 61094-3, "Measurement microphones, Part 3: Primary methods for free-field calibration of laboratory standard microphones by the reciprocity technique," 1995 .

${ }^{4}$ IEC International Standard 50(801), "International electrotechnical vocabulary," 1994.

"American National Standard ANSI S1.1, "Acoustical Terminology," 1994.

${ }^{6}$ F. Ingerslev, O. J. Pedersen, and P. K. Møller, "New rooms for acoustic measurements at the Danish Technical University," Acustica 19, 185-199 (1967/68).

${ }^{7}$ K. A. Cunefare, V. B. Biesel, J. Tran, R. Rye, A. Graf, M. Holdhusen, and A.-M. Albanese, "Anechoic chamber qualification: Traverse method, inverse square law analysis method, and nature of test signal," J. Acoust. Soc. Am. 113, 881-892 (2003).

${ }^{8}$ W. R. MacLean, “Absolute measurement of sound without a primary standard," J. Acoust. Soc. Am. 12, 140-146 (1940).

${ }^{9} \mathrm{~W}$. Wathen-Dunn, "On the reciprocity free-field calibration of microphones," J. Acoust. Soc. Am. 21, 542-546 (1949).

${ }^{10}$ L. L. Foldy and H. Primakoff, "A general theory of passive linear electroacoustic transducers and the electroacoustic reciprocity theorem. I," J. Acoust. Soc. Am. 17, 109-120 (1945).

${ }^{11} \mathrm{H}$. Primakoff and L. L. Foldy, "A general theory of passive linear elec- troacoustic transducers and the electroacoustic reciprocity theorem. II," J. Acoust. Soc. Am. 19, 50-58 (1947).

${ }^{12} \mathrm{~F}$. M. Wiener, "On the relation between the sound fields radiated and diffracted by plane obstacles," J. Acoust. Soc. Am. 23, 697-700 (1951).

${ }^{13}$ J. R. Cox, Jr., "Physical limitations on free-field microphone calibration," Massachusetts Institute of Technology, Ph.D. thesis, 1954.

${ }^{14} \mathrm{~K}$. Rasmussen, Acoustic centre of condenser microphones, The Acoustics Laboratory, Technical University of Denmark, Report No. 5, 1973.

${ }^{15}$ IEC Publication 486, "Precision method for free-field calibration of oneinch condenser microphones by the reciprocity technique," 1974.

${ }^{16}$ G. R. Hruska and W. Koidan, "Free-field method for sound-attenuation measurement," J. Acoust. Soc. Am. 58, 507-509 (1975).

${ }^{17}$ K. Rasmussen and E. Sandermann Olsen, "Intercomparison on free-field calibration of microphones," The Acoustics Laboratory, Technical University of Denmark, Report PL-07, 1993.

${ }^{18} \mathrm{P}$. M. Juhl, "A numerical investigation of standard condenser microphones," J. Sound Vib. 177, 433-446 (1994).

${ }^{19}$ R. P. Wagner and V. Nedzelnitsky, "Determination of acoustic center correction values for type LS2aP microphones at normal incidence," J. Acoust. Soc. Am. 104, 192-203 (1998).

${ }^{20}$ Y. Ando, "Experimental study of the pressure directivity and the acoustic centre of the 'circular pipe horn loud speaker," " Acustica 20, 366-369 (1968).

${ }^{21}$ W. James Trott, "Effective acoustic center redefined," J. Acoust. Soc. Am. 62, 468-469 (1977)

${ }^{22} \mathrm{M}$. Vorländer and H. Bietz, "Novel broad-band reciprocity technique for simultaneous free-field and diffuse-field microphone calibration," Acustica 80, 365-377 (1994).

${ }^{23}$ American National Standard ANSI S1.10-1966, "Method for calibration of microphones," 1966.

${ }^{24}$ S. Barrera Figueroa, New methods for transducer calibration: Free-field reciprocity calibration of condenser microphones, Acoustic Technology, Ørsted•DTU, Technical University of Denmark, Ph.D. thesis, 2003.

${ }^{25} \mathrm{P}$. M. Morse and K. U. Ingard, Theoretical Acoustics (McGraw-Hill, New York, 1968/1984). See Sec. 7.2.

${ }^{26}$ Handbook of Mathematical Functions, edited by M. Abramowitz and I. A. Stegun (National Bureau of Standards, Washington, DC, 1965).

${ }^{27}$ A. Papoulis, Signal Analysis (McGraw-Hill, New York, 1977). See Sec. 4-2.

${ }^{28}$ J. L. Leander, "On the relation between the wavefront speed and the group velocity concept," J. Acoust. Soc. Am. 100, 3503-3507 (1996)

${ }^{29}$ S. Barrera-Figueroa, K. Rasmussen, and F. Jacobsen, "A time selective technique for free-field reciprocity calibration of condenser microphones," J. Acoust. Soc. Am. 114, 1467-1476 (2003). 